\title{
Analysis of plasma waves observed in the inner Saturn magnetosphere
}

\author{
J. D. Menietti ${ }^{1}$, O. Santolik ${ }^{2}$, A. M. Rymer ${ }^{3}$, G. B. Hospodarsky ${ }^{1}$, D. A. Gurnett ${ }^{1}$, and A. J. Coates ${ }^{4}$ \\ ${ }^{1}$ Dept. of Physics and Astronomy, University of Iowa, Van Allen Hall, Iowa City, IA, USA \\ ${ }^{2}$ Inst. of Atmospheric Physics, ASCR, and Charles University, Prague, Czech Republic \\ ${ }^{3}$ Applied Physics Laboratory, Johns Hopkins University, Laurel, MD, USA \\ ${ }^{4}$ Mullard Space Science Lab., University College London, Dorking, UK
}

Received: 21 March 2008 - Accepted: 23 July 2008 - Published: 9 September 2008

\begin{abstract}
Plasma waves observed in the Saturn magnetosphere provide an indication of the plasma population present in the rotationally dominated inner magnetosphere. Electrostatic cyclotron emissions often with harmonics and whistler mode emission are a common feature of Saturn's inner magnetosphere. The electron observations for a region near $5 R_{S}$ outside and near a plasma injection region indicate a cooler low-energy $(<100 \mathrm{eV})$, nearly isotropic plasma, and a much warmer $(E>1000 \mathrm{eV})$ more pancake or butterfly distribution. We model the electron plasma distributions to conduct a linear dispersion analysis of the wave modes. The results suggest that the electrostatic electron cyclotron emissions can be generated by phase space density gradients associated with a loss cone that may be up to $20^{\circ}$ wide. This loss cone is sometimes, but not always, observed because the field of view of the electron detectors does not include the magnetic field line at the time of the observations. The whistler mode emission can be generated by the pancake-like distribution and temperature anisotropy $\left(T_{\perp} / T_{||}>1\right)$ of the warmer plasma population.
\end{abstract}

Keywords. Magnetospheric physics (Planetary magnetospheres; Plasma waves and instabilities) - Space plasma physics (Wave-particle interactions)

\section{Introduction}

Plasma waves in Saturn's inner magnetosphere commonly include whistler mode emission and chorus emission as well as electron cyclotron emission with often one or more harmonics (cf. Gurnett et al., 2005; Hospodarsky et al., 2008). Menietti et al. (2008) have recently reported plasma waves within plasma injection regions that are frequently observed within $15 R_{S}$ of Saturn. These authors report intense cho-

Correspondence to: J. D. Menietti

(john-menietti@uiowa.edu) rus and electron cyclotron (EC) waves with many harmonics. The plasma injection regions are believed to be a result of the rotationally-driven interchange instability (cf. Hill et al., 1981, 2005; Burch et al., 2005; Rymer et al., 2007, 2008). The electron distribution observed within these regions as observed in the inner magnetosphere consists of a tenuous warm plasma with $T_{\perp} / T_{\|}>1$ and electron phase space density peaked near a pitch angle of $90^{\circ}$ (pancake) and a much cooler and more isotropic distribution (cf. Andre et al., 2007; Rymer et al., 2008). These two distinct populations are consistent with a scenario of warm, tenuous, distant plasma contained in magnetic flux tubes that are adiabatically transported inward to a local much cooler population.

Outside and adjacent to the injection regions the observed particle distribution typically consists of a cool, low-energy, more field-aligned population and a warmer, higher-energy, more pancake or butterfly population (distributions peaked near pitch angles of $45^{\circ}$ and $135^{\circ}$ ). Burch et al. (2007) have suggested that outward flowing cool plasma has a source near the orbits of Tethys and Dione. Rymer et al. (2008) have the view that inward propagating tenuous, warm flux tubes can mix with the outward cool flux tubes to produce the observed butterfly distributions. Intense plasma waves observed in the inner magnetosphere can give clues to not only the observed plasma population, but also "hidden" plasma at pitch angles and/or energies beyond the limits of the electron low-energy spectrometer (ELS). Electrostatic cyclotron harmonic $(\mathrm{ECH})$ emissions at frequencies near the electron cyclotron frequency, $f_{c e}$, and $(n+1 / 2) f_{c e}$ are typically observed in the terrestrial magnetosphere near the magnetic equator and interact strongly with $\mathrm{keV}$ electrons (Horne and Thorne, 2000). Ashour-Abdalla and Kennel (1978) thoroughly discuss the role of loss cones in the generation of ECH emissions for a range of plasma parameters, loss cone widths and depths. Horne and Thorne (2000) and Horne et al. (2003) have shown the effectiveness of "pancake" electron phase space distributions (peaked at large pitch angles)

Published by Copernicus Publications on behalf of the European Geosciences Union. 
and temperature anisotropies $\left(T_{\perp} / T_{\|}>1\right)$ in exciting whistler mode and chorus emission at Earth; and of narrow (only a few degrees) loss cones in generating ECH emissions. These latter authors argue that, for the terrestrial case, ECH waves scatter electrons into the loss cone to form the observed pancake distributions. Glauert and Horne (2005) discuss how whistler mode emission can essentially increase the width of a loss cone as a result of pitch angle scattering that is effective at larger pitch angle.

At Earth, intense, diffuse whistler mode hiss at frequencies typically less than a few $\mathrm{kHz}$ is observed within the plasmasphere and outside the plasmapause and is generally believed to be generated by a temperature anisotropy in the electron phase space distribution. Natural emissions of discrete, whistler-mode chorus at Earth consist of electromagnetic waves in the frequency range from a few hundred hertz to greater than $10 \mathrm{kHz}$ observed in the lower density region outside the plasmapause. These emissions are most often characterized as narrow-banded at $f<f_{c e} / 2$ and/or $f>f_{c e} / 2$ with an emission gap near $f_{c e} / 2$. It is generally believed that chorus is generated by a nonlinear process based on the electron cyclotron resonance of whistler-mode waves with energetic electrons, taking place close to the geomagnetic equatorial plane (Omura et al., 1991; Nunn et al., 1997; LeDocq et al., 1998). Storm-time chorus is especially important for the physics of the Earth's magnetosphere since it can significantly influence the distribution functions of the energetic electrons in the outer radiation belt (e.g. Meredith et al., 2003; Horne et al., 2003; Horne and Thorne, 2003). Observations of chorus emission at Saturn have recently been reported by Hospodarsky et al. (2008). In the present paper we report on the plasma and wave observations typically observed in the inner magnetosphere for $r \lesssim 8 R_{S}$ and outside plasma injection regions. The specific time intervals of the wave data were chosen based on these criteria and on the availability of electron phase space density distributions. Long-term periodicities of the emission as discussed by Louarn et al. (2007) are not considered in this paper. We seek to explain the free energy sources of the observed plasma waves. We model the electron distribution function to show that EC emissions similar to those observed can be generated by a loss cone that is implicated in the data. Whistler mode emissions at frequencies similar to the observed emissions can be excited by the observed pancake distribution with a temperature anisotropy.

\section{Instrumentation}

\subsection{RPWS}

The Cassini Radio and Plasma Wave Science (RPWS) instrument measures oscillating electric fields over the frequency range $1 \mathrm{~Hz}$ to $16 \mathrm{MHz}$ and magnetic fields in the range $1 \mathrm{~Hz}$ to $12 \mathrm{kHz}$ (cf. Gurnett et al., 2004). The instrument uses 3 nearly orthogonal electric field antennas and 3 orthogonal magnetic search coil antennas, providing a direction-finding capability. There are 5 receiver systems: the high frequency receiver (HFR) covering $3.5 \mathrm{kHz}$ to $16 \mathrm{MHz}$; the medium frequency receiver (MFR) covering $24 \mathrm{~Hz}$ to $12 \mathrm{kHz}$; a low frequency receiver (LFR) covering $1 \mathrm{~Hz}$ to $26 \mathrm{~Hz}$; a 5 -channel waveform receiver which operates in either a $1-26 \mathrm{~Hz}$ or $3 \mathrm{~Hz}-2.5 \mathrm{kHz}$ mode; and a high resolution wideband receiver (WBR) that covers two frequency bands, $60 \mathrm{~Hz}$ to $10.5 \mathrm{kHz}$ and $800 \mathrm{~Hz}$ to $74 \mathrm{kHz}$. The data presented in this study are measured by the LFR, MFR, and WBR.

\subsection{ELS}

The Cassini Plasma Spectrometer (CAPS) is composed of three sensors: the electron spectrometer (ELS), the ion beam spectrometer (IBS), and the ion mass spectrometer (IMS). Of importance in this study is the ELS which contains an 8 -detector fan array in a single plane. Each detector has a $5.2^{\circ} \times 20^{\circ}$ field of view for a total in-plane field of view of $160^{\circ}$. The instrument measures electron energy from $0.6 \mathrm{eV}$ to $28250 \mathrm{eV}$ over a period of $2 \mathrm{~s}$ with a resolution of $\Delta E / E=0.17$ (cf. Young et al., 2004).

\section{Observations and wave growth rate analysis}

In Fig. 1 we display a frequency-time spectrogram of electric field data from the low-rate wave receiver with plasma wave intensity color-coded in decibels and a linear frequency scale. The plot covers a two-hour period from 30 October 2005, and orbital position parameters are shown at the bottom of the plot. The spectrogram shows electron cyclotron (EC) waves and harmonics above the electron cyclotron frequency (lower white line), and whistler mode/chorus emission for $f<f_{c e}$. During this period we see that the ECH emissions vary in intensity and the fundamental emission is usually significantly stronger than the harmonics, which are often missing. The whistler mode emission for $f \gtrsim 500 \mathrm{~Hz}$ varies in bandwidth and also in intensity.

During portions of this time interval high resolution wideband receiver data are also available, and we display a four minute interval from 03:13-03:17 in Fig. 2. In this plot the frequency range is now linear and the white line again indicates the electron cyclotron frequency. This plot ranges to a maximum frequency of $10 \mathrm{kHz}$ (maximum range for the mode of the spacecraft at this time) and the intensities are given in absolute scale of $V^{2} /\left(m^{2} \mathrm{~Hz}\right)$. The most intense fundamental emission extends from about $3.8 \mathrm{kHz}$ to perhaps $5.8 \mathrm{kHz}$. The first harmonic emission is up to an order of magnitude less intense at places with the strongest emission extending in frequency from about $8.1 \mathrm{kHz}$ to about $9.5 \mathrm{kHz}$.

Whistler mode emission is dominant at frequencies less than $f_{c e}$ with a maximum frequency of about $1.4 \mathrm{kHz}$ and extending down below $500 \mathrm{~Hz}$ where spacecraft interference 


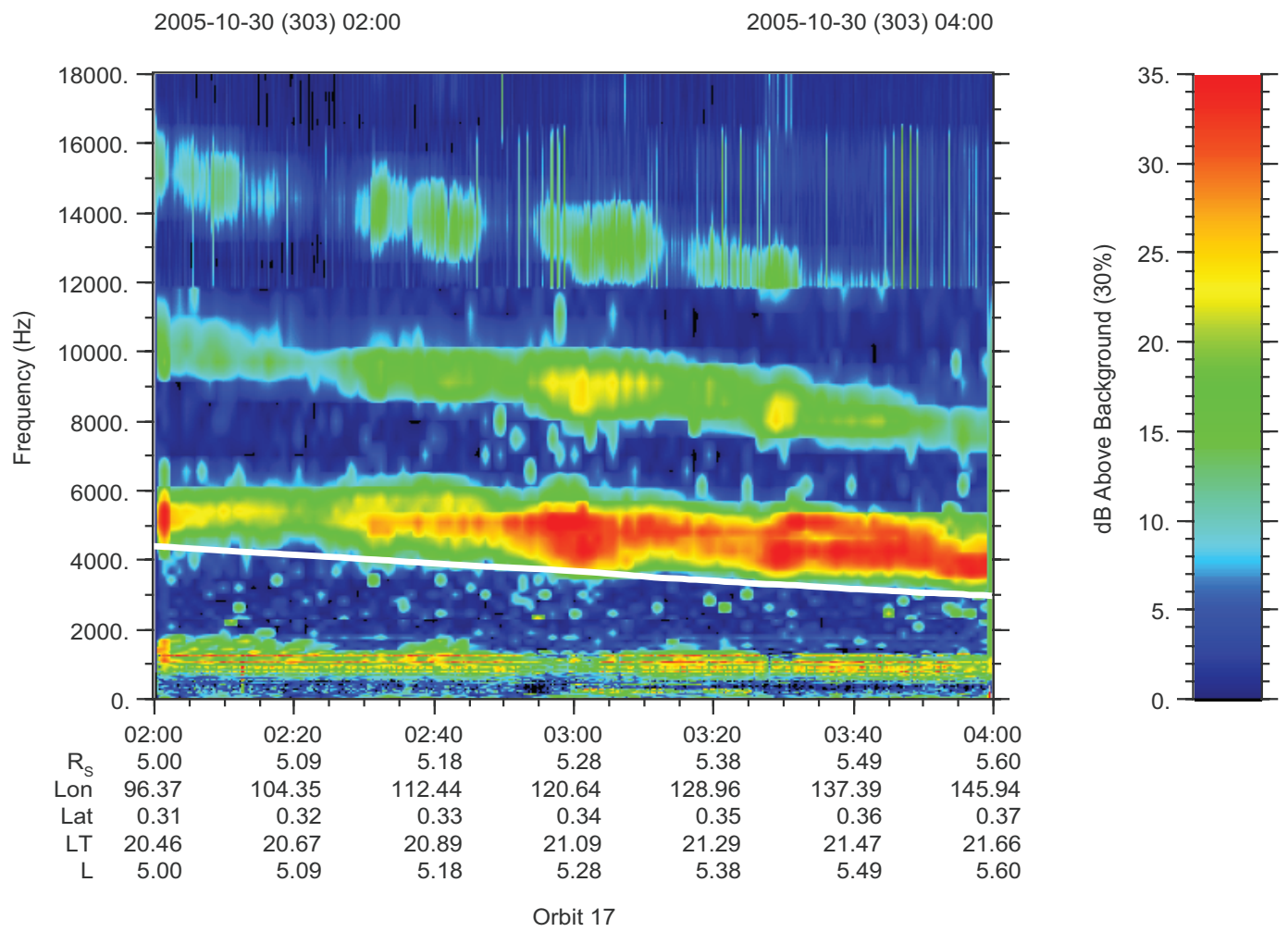

Fig. 1. A frequency-vs-time spectrogram with relative intensity color-coded in decibels and a linear frequency scale. The plot covers a two-hour period from 30 October 2005, and the data are from the low-rate wave receiver. The white line indicates the electron cyclotron frequency. Orbital position parameters are shown at the bottom of the plot.

Table 1. Plasma distribution fitting parameters for time interval 1.

\begin{tabular}{lcccccc}
\hline & Density $(\% *)$ & $W_{\|}(\mathrm{eV})$ & $T_{\perp} / T_{\|}$ & $V_{d}$ & $\Delta$ & $\beta$ \\
\hline Cold background & 5.0 & 0.053 & 1.0 & 0 & 0.85 & 0.7 \\
Cool core & 94.0 & 4.73 & 1.318 & 0 & 0.85 & 0.7 \\
Warm & 1.0 & 1184 & 1.963 & 0 & 0.85 & 0.35 \\
Cold ions & 100 & - & - & - & - & - \\
\hline
\end{tabular}

*Total density is $49.4 \mathrm{~cm}^{-3}$

bands are encountered. We analyzed the waveform receiver data for $f<2 \mathrm{kHz}$ obtained at this time, using the Means method (Means, 1972; cf. LeDocq et al., 1998). In Fig. 3 we display an analysis spectrogram of the 5-channel waveform receiver data showing the electric and magnetic fields as well as the wave normal directions and the wave direction relative to the magnetic field line for a 4-min interval of the whistler mode emission observed. The panels contain (top to bottom) electric field; magnetic field; wave normal angle $(\theta)$ and azimuthal angle $(\phi)$ relative to the magnetic field; the Poynting vector angle, $\theta_{s}$; parallel component of the Poynting vector $\boldsymbol{S}$ relative to the static magnetic field $\boldsymbol{B}$ (red is toward the magnetic equator). The calculated wave normal angle displayed in panel 3 indicates values nearly aligned or anti-aligned with the magnetic field. In the bottom panel we see that for $f>500 \mathrm{~Hz}$ (above interference lines) the dominant wave direction is toward the equator. The polarization of the emission (not shown) is right hand circular, consistent with the whistler mode.

We have fitted the observed ELS electron distribution function contours using a sum of bi-Maxwellians as follows

$$
\begin{aligned}
f_{S}\left(v_{\perp}, v_{\|}\right) & =\sum_{S}\left(\frac{n_{s}}{\pi^{\frac{3}{2}} w_{\perp_{s}}^{2} w_{\|_{s}}}\right)\left[\left(1-\Delta_{s}\right) e^{-\frac{v_{\perp}^{2}}{w_{\perp s}^{2}}}\right. \\
& +\frac{\Delta_{s}}{\left(1-\beta_{s}\right)} \cdot\left(e^{\left.\left.-\frac{v_{\perp}^{2}}{w_{\perp s}^{2}}-e^{-\frac{v_{\perp}^{2}}{\beta_{s} w_{\perp s}^{2}}}\right)\right] e^{-\left(\frac{\left.v_{\|}-v_{d s}\right)^{2}}{w_{\|_{s}}^{2}}\right)}}\right.
\end{aligned}
$$



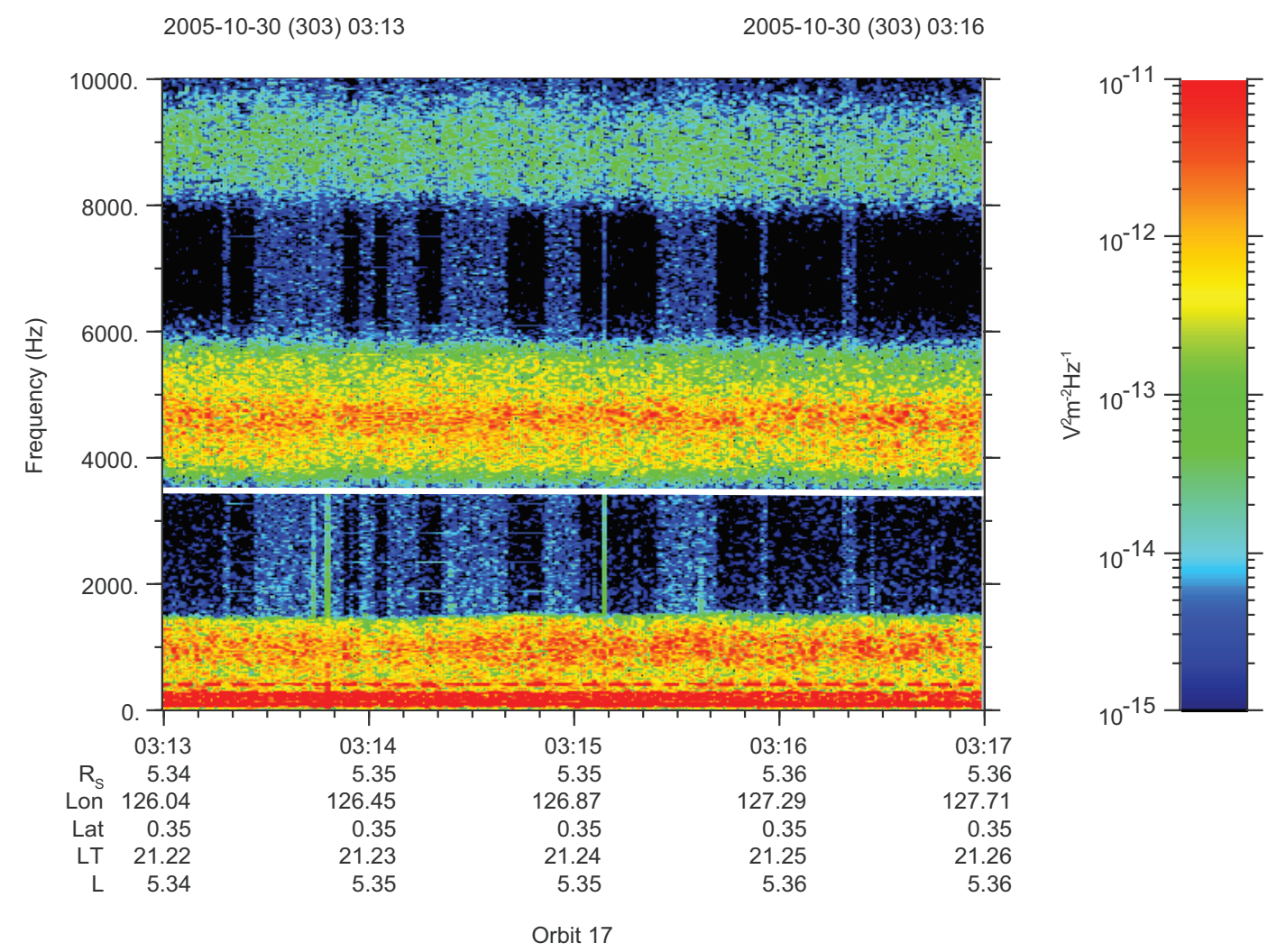

Fig. 2. A spectrogram of high resolution wideband receiver data for a five minute interval from 03:13-03:17 from Fig. 1. The frequency range is now linear and the white line shows the electron cyclotron frequency.

where $v_{\|}$and $v_{\perp}$ are the particle velocities parallel and perpendicular to the magnetic field, respectively; $w_{\|}$and $w_{\perp}$ are the parallel and perpendicular thermal velocities, respectively; $v_{d}$ is the parallel drift velocity. The parameters $\Delta$ and $\beta$ describe the depth and width of the model loss cone, respectively. The sum of Maxwellians, with large number of fitting parameters, provides a good fit to the data as will be shown.

To investigate the source of these waves (Figs. 1-3) we require the electron phase space distribution. In Fig. 4a, b we display contours of the electron distribution measured by ELS for energies less than $18 \mathrm{keV}$ and $70 \mathrm{eV}$, respectively, during the time range 03:15:20 to 03:15:30 (which we refer to as interval 1) within the time interval shown in Fig. 2. This is a time period outside of any plasma injection region where we observe an intense EC fundamental emission but weaker harmonics, and strong whistler mode emission. The higher energy and warmer plasma distribution appears somewhat pancake $\left(T_{\perp} / T_{\|}>1\right)$ with an indication of a loss cone for electrons nearly aligned with the magnetic field. Note that, because of the spacecraft orientation at the time, there are no electron measurements at pitch angles within about $20^{\circ}$ of the field line. For lower energy electrons Fig. $4 \mathrm{~b}$ shows a more isotropic distribution, and again there is an indication of a loss cone seen for electrons at the smallest pitch angles. We use a non-linear least squares fitting routine to fit the model distribution, Eq. (1), to the data. We have assumed four populations of plasma, three electron distributions and a cold plasma ion distribution. The electron populations include a dominant cool core plasma, a low-density higher-energy warm plasma, and a very cold isotropic electron background plasma. The ions are assumed to be a cold plasma background. We initially adjusted and fixed the thermal velocity and relative density of the cold background plasma (which has an energy less than the lowest energy of the ELS) to a value which allowed the bandwidth of the fundamental ECH band to be close to that observed. The total density of the plasma was fixed by observations of the local upper hybrid resonance at the time, $f_{u h}=63.3 \mathrm{kHz}$ (not shown in Fig. 1). In Table 1 we present the fitting parameters determined for time interval $1,03: 15: 20$ to $03: 15: 30$.

\subsection{ECH emissions}

The loss cone of the cool core distribution is critical for the generation of the EC and harmonic emissions. The higher energy pancake distribution and the temperature anisotropy are responsible for the growth of the whistler-mode/chorus emissions at lower frequency. To estimate the size of an expected loss cone near the magnetic equator we note that Saturn 


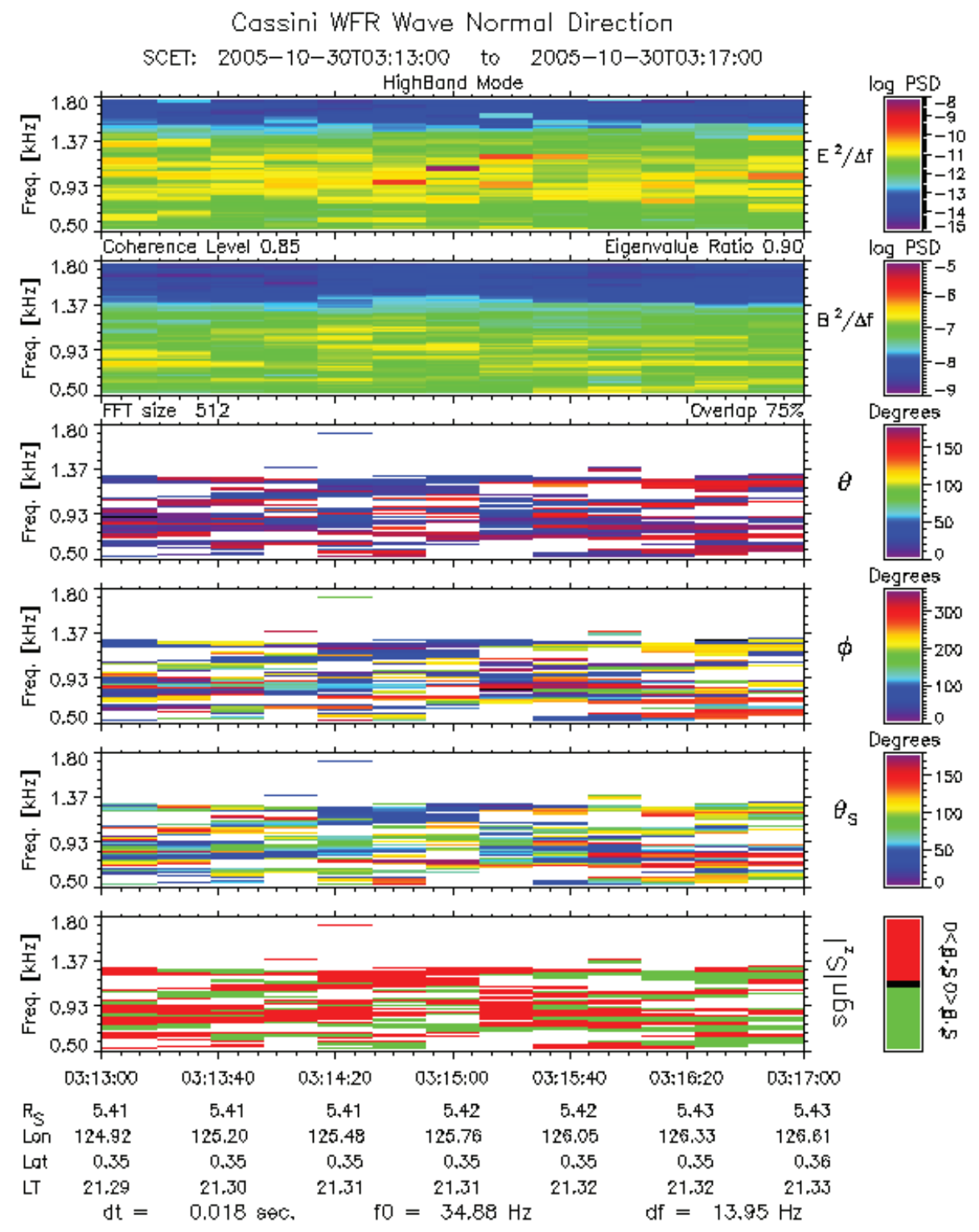

Fig. 3. An analysis spectrogram of the 5 -channel waveform receiver data for $500 \mathrm{~Hz}<f<1800 \mathrm{~Hz}$ showing the electric and magnetic fields as well as the wave normal directions within interval 1. The panels contain (top to bottom) electric field; magnetic field; wave normal angle $(\theta)$ and azimuthal angle $(\phi)$ relative to the magnetic field; the Poynting vector angle, $\theta_{s} ;$ and $\boldsymbol{S} \cdot \boldsymbol{B}$ (red is toward the magnetic equator).

kilometric radio emissions are observed up to $\sim 1 \mathrm{MHz}$. This emission is believed to be due to the cyclotron maser instability and requires a source of electrons. Assuming a magnetic field as given by Connerney et al. (1983), and assuming a mirror point near an attitude in the polar region where $f_{c e} \sim 1 \mathrm{MHz}$ we obtain a loss cone of less than $\sim 5$ degrees for $L=5.35$, near the position of the observed plasma injection. Such a loss cone would not be visible to the ELS instrument during the time of the observations. We found that introducing such a narrow loss cone leads to strong EC wave growth and strong first and second harmonic emission, contrary to the observations at this time which show a weaker first har- monic and very weak second harmonic. For this reason we increased the width of the model loss cone to $\sim 20$ degrees, which is also consistent with the observations which suggest a loss cone at the smallest observed pitch angles near $20^{\circ}$. In Fig. 5a we overplot contours of the model electron distribution function and the actual data, and in Fig. $5 \mathrm{~b}$ we show contours of the model distribution including a loss cone.

To determine roots of the dispersion equation and to calculate the growth rate of the plasma waves resulting from the model distribution function, we have used a modification of a warm plasma dispersion solver (cf. Santolik and Parrot, 1996), based on the susceptibility tensor calculated 

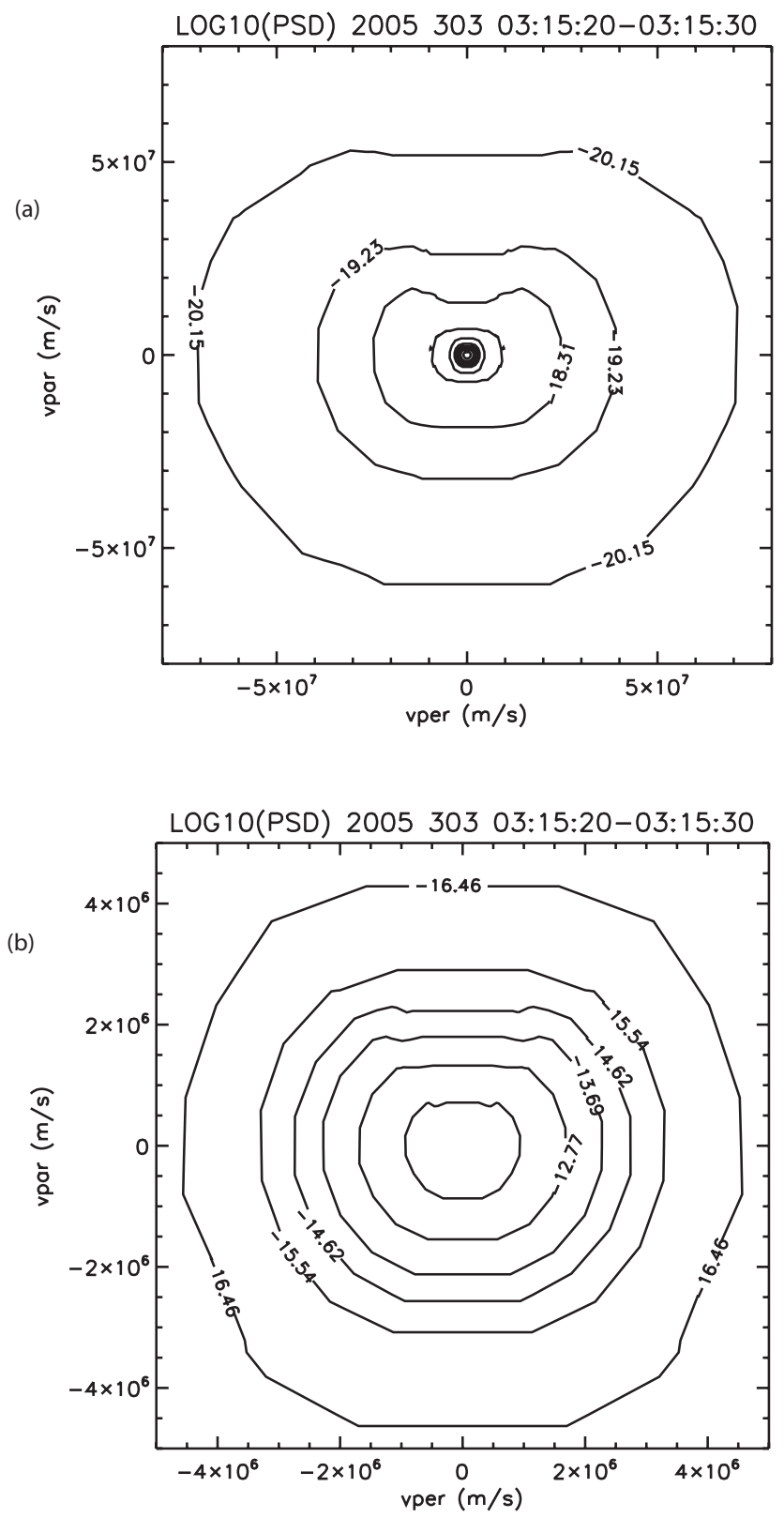

Fig. 4. Contours of the electron phase space density $\left(\mathrm{s}^{3} / \mathrm{m}^{6}\right)$ observed by ELS for energies less than $18 \mathrm{keV}$ (a) and $70 \mathrm{eV}$ (b), during the time interval 03:15:20 to 03:15:30 (time interval 1).

by the program, Waves in Homogeneous, Anisotropic Multicomponent Plasmas (WHAMP) (cf. Ronnmark, 1982, 1983). The solver also includes a cold plasma susceptibility tensor, which we used for the background ion component. In Fig. 6 we display the results for the EC emissions near maximum growth rate at the fundamental and first harmonic frequencies and wave normal angles $84.2^{\circ}$ and $88.5^{\circ}$, respectively. Emission at the second harmonic produced almost no growth. This plot shows the real frequency versus wave number in the lower panel and the imaginary frequency relative to $f_{c e}$ in the
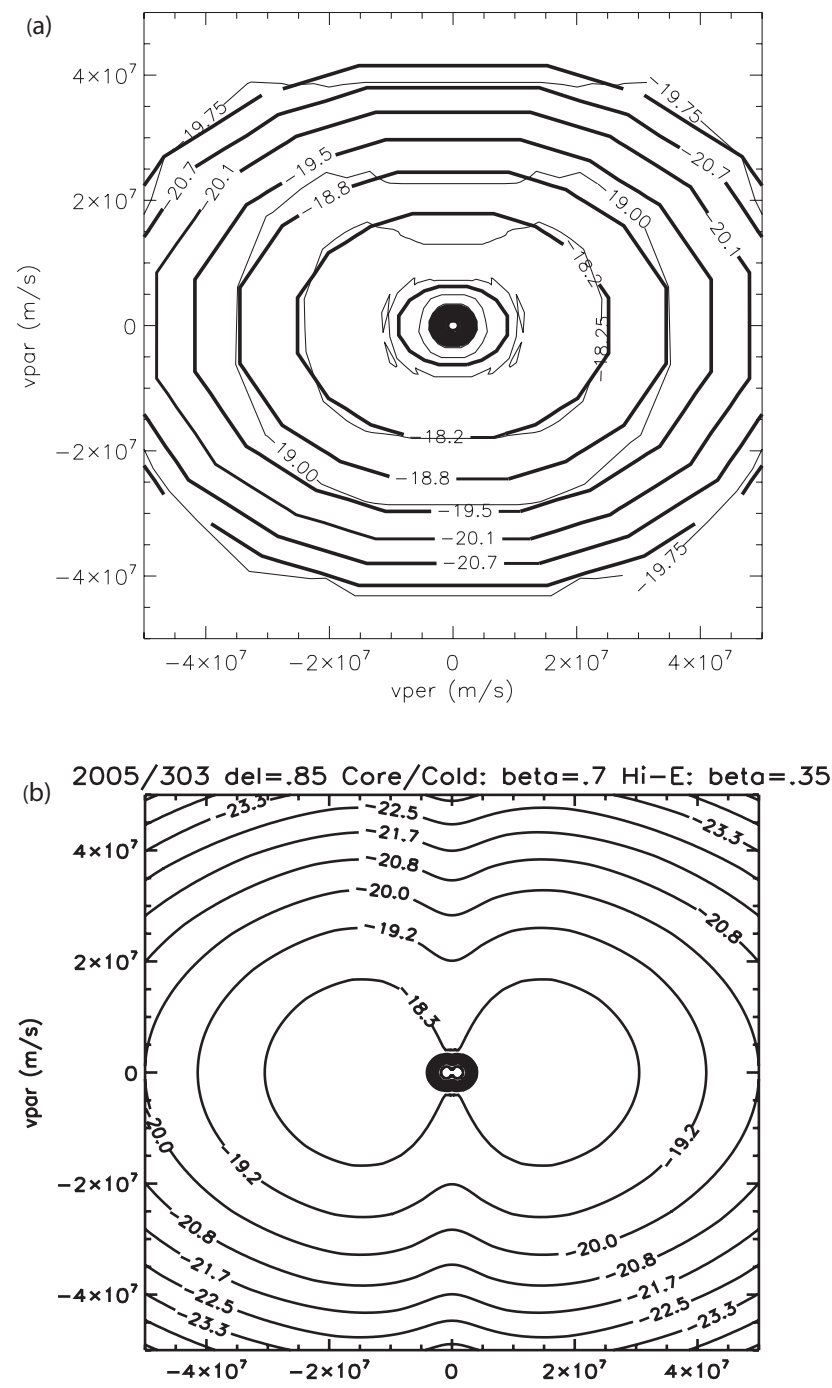

Fig. 5. An overplot of contours of the model electron distribution function (bold) and the actual data (a). In panel (b) we show the model contours including the loss cone, which is essentially invisible to the ELS detectors.

upper panel. In Fig. 7 we show emission at the fundamental for a range of wave normal angles $85^{\circ}<\theta<88^{\circ}$. Emission at the first harmonic showed growth only over a very narrow range of wave normal angles. The wave normal angle that produced the largest growth rate for the fundamental (first harmonic) emission was $84.2^{\circ}\left(88.45^{\circ}\right)$. The maximum growth is $\sim 0.052 f_{c e}$ for $f_{\text {real }}=5359 \mathrm{~Hz}$ at the fundamental with a bandwidth extending from $\sim 4000 \mathrm{~Hz}$ to $\sim 5500 \mathrm{~Hz}$ over a range of wave normal angles $\sim 84.1^{\circ}<\theta<88.1^{\circ}$. The observed emission near the fundamental appears to extend from just above $f_{c e}$ at $\sim 3600 \mathrm{~Hz}$ and drops off rapidly above $\sim 5900 \mathrm{~Hz}$, which is a somewhat larger bandwidth than modeled. For the first harmonic emission the maximum growth is $0.026 f_{c e}$ for $f_{\text {real }}=9172 \mathrm{~Hz}$ with a bandwidth extending from $\sim 7300 \mathrm{~Hz}$ to $\sim 9200 \mathrm{~Hz}$ over a much narrower range of wave 

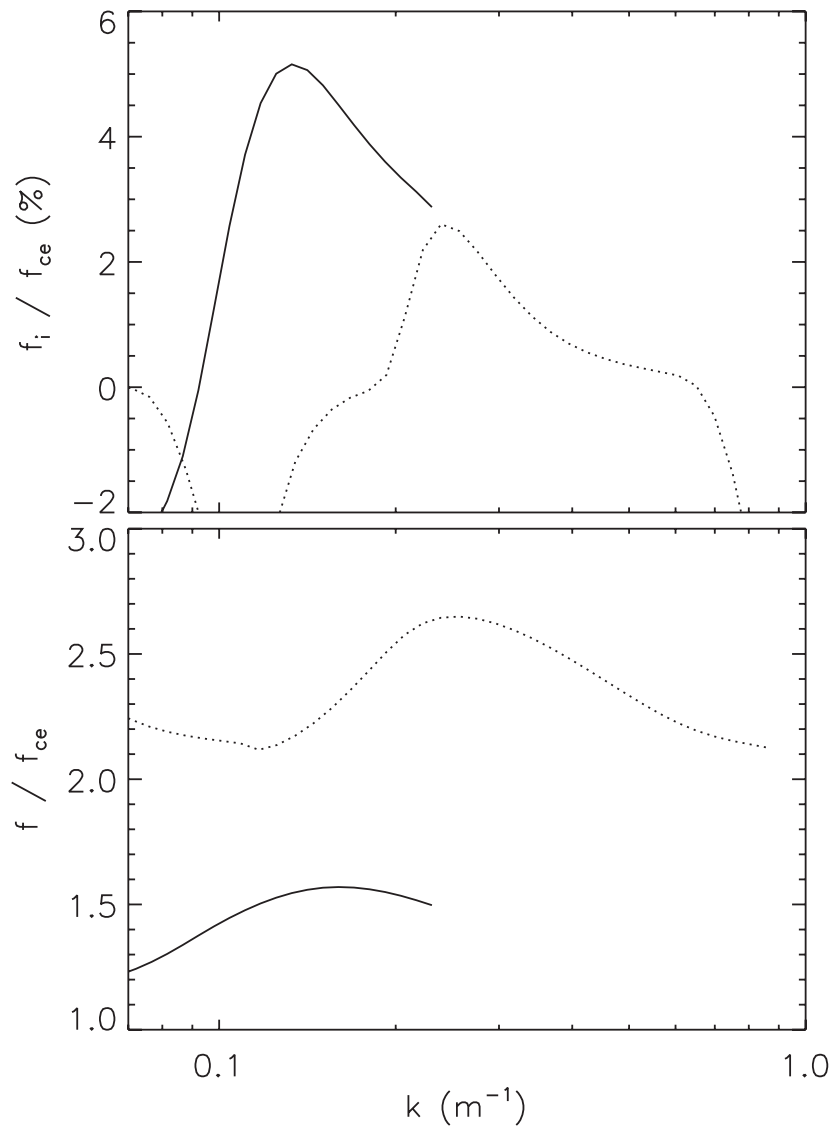

Fig. 6. Results for the dispersion and growth rate analysis for the EC emissions at the fundamental (solid) and first harmonic (dotted) frequencies. Emission at the second harmonic produced almost no growth. The lower panel shows the real frequency versus wave number and the imaginary frequency relative to $f_{c e}$ is in the upper panel.

normal angles, $88.45<\theta<89.6^{\circ}$. The observed bandwidth for the more intense emission extends from about $8000 \mathrm{~Hz}$ to about $9500 \mathrm{~Hz}$, with the center of the peak near $8870 \mathrm{kHz}$. The modeled emission is shifted to lower frequency by several hundred $\mathrm{Hz}$, but does substantially overlap the observed emission. Growth of the ECH emission is dependent on the velocity space density gradient provided by the loss cone. The pancake distribution at higher energies without the loss cone is insufficient to drive the $\mathrm{ECH}$ emission.

We can estimate the signal gain produced by these growth rates. From the calibrated observations we estimate the maximum signal strength of the ECH emission to be about $30 \mathrm{~dB}$ above background for the fundamental and about $20 \mathrm{~dB}$ for the first harmonic. From the growth rate calculations of the fundamental emission shown above the group velocity of the wave is about $3.8 \times 10^{-4} c$ and $\omega_{i} \sim 0.03 \omega_{c e}$ so the path length required to produce $30 \mathrm{~dB}$ of gain is $\sim 1 \mathrm{~km}$. This is quite short. Saturation of this emission could be accomplished by pitch angle scattering and filling of the loss cone

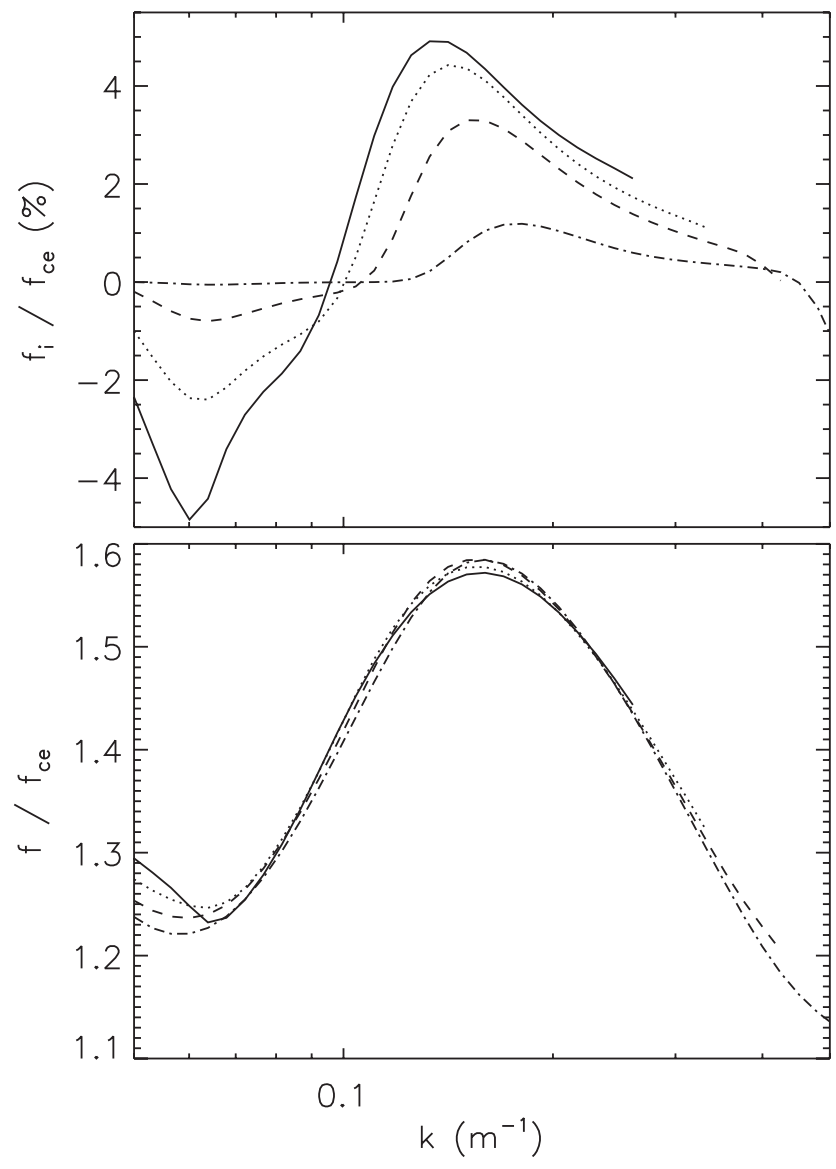

Fig. 7. EC emission at the fundamental for a range of wave normal angles $85^{\circ}$ (solid), $86^{\circ}$ (dotted), $87^{\circ}$ (dashed), and $88^{\circ}$ (dot-dash). Emission at the first harmonic showed growth only over a very narrow range of wave normal angles.

as discussed by Horne et al. (2003). The latter authors have modeled pitch angle scattering due to $\mathrm{ECH}$ waves for a terrestrial substorm. They find that these waves are very effective at scattering electrons into the loss cone, and provide the major contribution to diffuse auroral precipitation for a substorm event observed near the magnetic equator. The diffusion time was found to be comparable to the duration of the event, consistent with the theory that filling of the loss cone by pitch angle scattering can saturate the wave growth.

\subsection{Whistler mode emissions}

The same electron distribution function shown in Fig. 5b is also unstable to the growth of whistler mode emission at small wave normal angles. The critical parameters include both the magnitude of the temperature anisotropy and the wave normal angle, $\theta$. The free energy source is the higher energy, pancake distribution. We have calculated the growth rates for whistler emission again using the distribution function fitting parameters of Table 1 . In Fig. 8 we plot the real (imaginary) frequencies as before in the bottom (upper) 

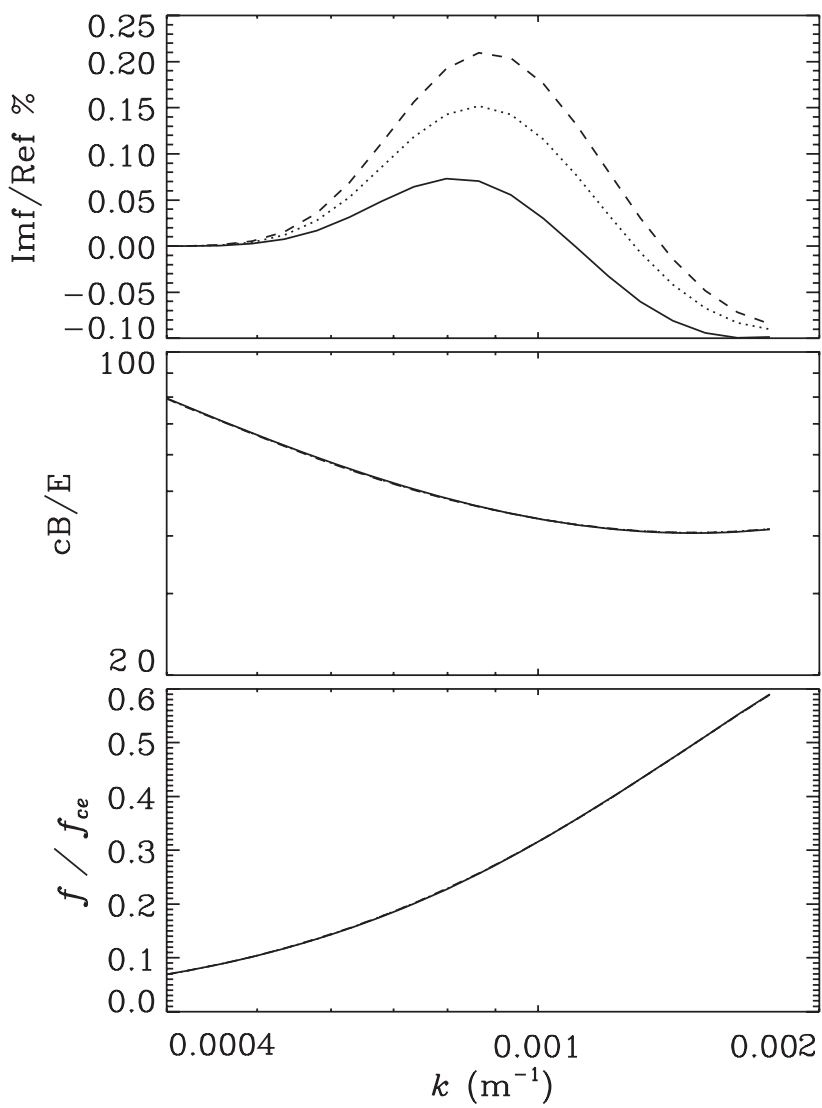

Fig. 8. The real (imaginary) frequencies in the bottom (upper) panels. The middle panel displays the index of refraction as $c B / E$. The whistler mode emission is seen to grow for frequencies in the approximate range $400 \mathrm{~Hz}<f<1300 \mathrm{~Hz}$ as observed. The growth rate is shown for the best fit value of $T_{\perp} / T_{\|}=1.96$ (solid) as well as $T_{\perp} / T_{||}=3$ (dotted) and $T_{\perp} / T_{\|}=4$ (dashed).

panels. The middle panel displays the index of refraction as $c B / E$. The whistler mode emission is seen to grow for frequencies in the approximate range $400 \mathrm{~Hz}<f<1300 \mathrm{~Hz}$, with positive growth rates for wave normal angles less than about 10 degrees. The model bandwidth agrees reasonably well with the observed frequency range of the whistler mode emission. We show the growth for the fitted value of $T_{\perp} / T_{\|}=1.96$ (solid). The peak growth rate is about $0.07 \%$ $f_{\text {real }}$ for $f_{\text {real }}=889 \mathrm{~Hz}$ and $\theta=0.1^{\circ}$. The peak growth rate decreases for larger $\theta$ up to about $10^{\circ}$. Also shown are curves for arbitrarily chosen larger values of the temperature anisotropy for the higher energy pancake distribution only. We chose $T_{\perp} / T_{||}=3.06$ and $T_{\perp} / T_{||}=4.0$ showing the effect of this parameter on the growth rate. Note the growth rates are shown relative to the real frequency rather than $f_{c e}$ as for the $\mathrm{ECH}$ emissions. The growth is seen to increase significantly with increasing values of $T_{\perp} / T_{||}$. The wave normal angle was held constant at $0.1^{\circ}$ for each curve shown in Fig. 8 .
We estimate the signal gain produced by these whistler mode growth rates. The maximum signal strength for the whistler mode observations is also about $30 \mathrm{~dB}$ above background as determined from the calibrated measurements. The growth rates of the whistler mode vary from about $\omega_{i}=3.9 \mathrm{rad} / \mathrm{s}$ for the case of $T_{\perp} / T_{\|}=1.96$ to $\omega_{i}=12.8 \mathrm{rad} / \mathrm{s}$ for $T_{\perp} / T_{\|}=4.0$, so the required path length to obtain $30 \mathrm{~dB}$ of growth would range from about $5700 \mathrm{~km}$ to $18000 \mathrm{~km}$. This length is much larger than for the ECH emissions, however, the whistler mode emissions travel almost along the magnetic field line (instead of oblique to it as do the ECH emissions). If the size of the whistler mode generation region near the magnetic equator at Saturn is several thousand kilometers as it is at Earth for the chorus emission (cf. Parrot et al., 2003; Santolik et al., 2004), then it may be possible to attain gains of $30 \mathrm{~dB}$ for the whistler mode, but if the temperature anisotropy in the source region is larger than we found as a best fit to the locally measured electron distribution for interval 1 , the source region could be much smaller.

\subsection{Time interval $2(02: 58: 30-02: 58: 40)$}

We have also examined an earlier time interval on this day during which the whistler mode emissions are much weaker, while the ECH emissions are similar. In Fig. 9 we display a spectrogram of the high resolution wave data for the time interval 02:55 to 03:00. Note that the whistler mode emission for $f<f_{c e}$ is weaker than for interval 1. The banded emission for $f \lesssim 500 \mathrm{~Hz}$ is again contaminated with spacecraft interference. We concentrate on the interval 02:58:30-02:58:40 for which we have obtained the electron phase space distribution. The whistler mode emission extends from about $500 \mathrm{~Hz}$ to perhaps $1100 \mathrm{~Hz}$. The band of fundamental EC extends from about $f_{c e}(3725 \mathrm{~Hz})$ to over $6 \mathrm{kHz}$, but the most intense emission is in the band from about $4 \mathrm{kHz}$ to $5.2 \mathrm{kHz}$. The first harmonic emission extends from about $8.2 \mathrm{kHz}$ to about $9.9 \mathrm{kHz}$. Because of the operating mode at the time, the data were available only over a pitch angle range from $25.8^{\circ}<\alpha<99.6^{\circ}$. We have thus assumed not only gyrotropy with respect to the $V_{\|}$axis, but we also mirrored the electron distribution about the $V_{\perp}$ axis $\left(90<\alpha<180^{\circ}\right)$. We lend credence to this procedure by noting that during the nearby interval 02:55:00-02:55:10 the electron distribution was obtained only for the pitch angle range $80^{\circ}<\alpha<160^{\circ}$. This distribution was nearly a perfect mirror about the $V_{\perp}$ axis of the distribution shown in Fig. 10a. In Fig. 10a we plot contours of the observed distribution obtained by this method for $E<7 \mathrm{keV}$. Proceeding as before, we have fit the data to the functional form of Eq. (1) using the parameters listed in Table 2 . We again chose the density and temperature of the cold electron background by matching the frequency bandwidth of the fundamental EC emission as closely as possible. The total density was determined by observations of the upper hybrid resonance frequency at the time, $f_{u h}=65.2 \mathrm{kHz}$ (not shown). Figure 10b is a plot of contours of the phase 


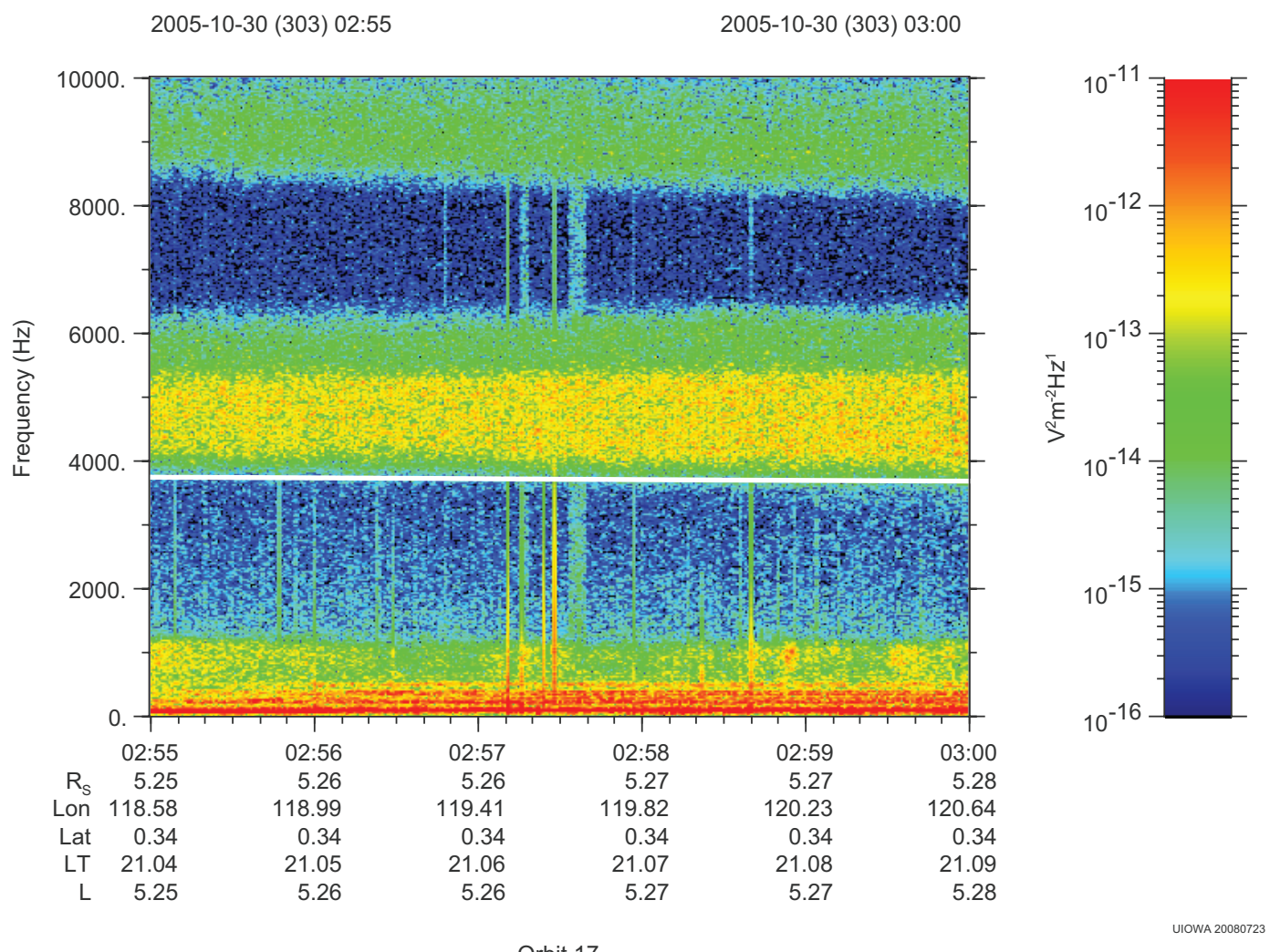

Fig. 9. A spectrogram of the high resolution wave data for the time interval 02:55 to 03:00. Note that the whistler mode emission for $f<f_{c e}$ is often weak. The banded emission for $f<500 \mathrm{~Hz}$ is contaminated with spacecraft interference. The white line indicates the electron cyclotron frequency.

Table 2. Plasma distribution fitting parameters for time interval 2.

\begin{tabular}{lcccccc}
\hline & Density $\left(\%^{*}\right)$ & $W_{\|}(\mathrm{eV})$ & $T_{\perp} / T_{\|}$ & $V_{d}$ & $\Delta$ & $\beta$ \\
\hline Cold background & 5.0 & 0.104 & 1.0 & 0 & 0.85 & 0.7 \\
Cool core & 94.0 & 6.27 & 1.098 & 0 & 0.85 & 0.7 \\
Warm & 1.0 & 1046 & 1.25 & 0 & 0.85 & 0.35 \\
Cold ions & 100 & - & - & - & - & - \\
\hline
\end{tabular}

*Total density is $52.48 \mathrm{~cm}^{-3}$

space density with an overlay of the fitted data. We have again assumed a loss cone with a width of about $20^{\circ}$ as before, but the model loss cone is not included in the overplot. In Fig. 11 we display the results of the growth rate analysis for the EC fundamental and first harmonic emissions in the same format as in Fig. 6. The curves are shown near maximum growth for $\theta=84.4^{\circ}$ (fundamental) and $\theta=87.8^{\circ}$ (first harmonic). The fundamental emission growth rate is about 3 times that of the first harmonic. In Fig. 12 we show the fundamental emission at 3 wave normal angles, $85^{\circ}, 86.5^{\circ}$, and $87.5^{\circ}$, displaying a range of frequencies and bandwidths over which wave growth occurs. From the calibrated observations of the fundamental EC emission, we estimate the typical gain to be 250 yielding a path length required to produce this gain of about $900 \mathrm{~m}$, similar to the length calculated for time interval 1 (near 03:15:20).

We have calculated the growth rates for whistler mode emission for interval 2 using the same distribution function fitting parameters of Table 2. In Fig. 13 we plot the real (imaginary) frequencies as before in the bottom (upper) panels. The middle panel displays the index of refraction as $c B / E$. The whistler mode emission is seen to grow weakly for frequencies in the approximate range $\sim 500 \mathrm{~Hz}<f<\sim 900 \mathrm{~Hz}$, for wave normal angles less than about 10 degrees. The model bandwidth agrees reasonably well with the observed frequency range of the whistler mode 

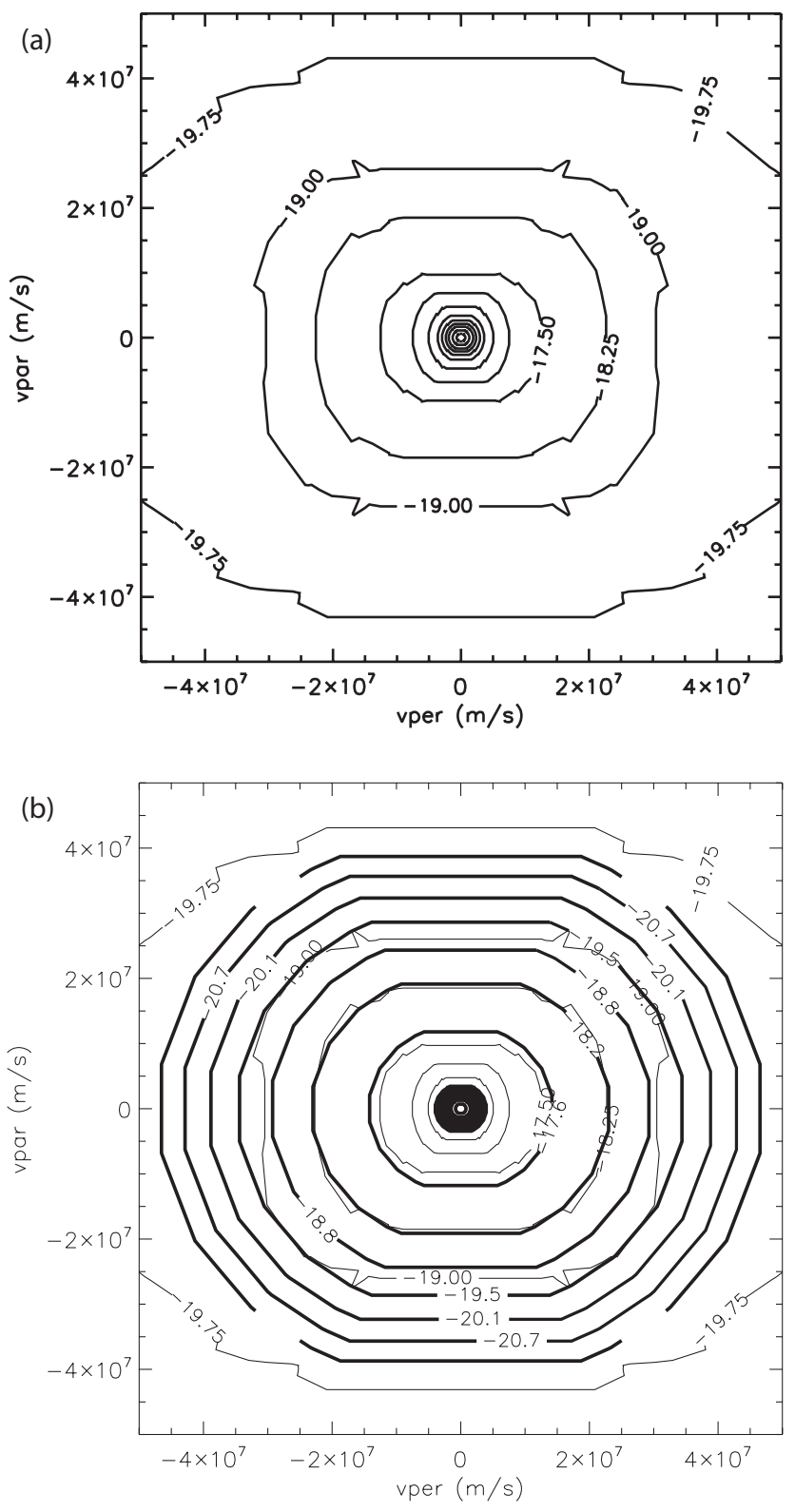

Fig. 10. (a) Contours of the observed phase space distribution for the time interval 2, 02:55:00-02:55:10, obtained as described by mirroring the data as described in the text. (b) An overplot of the contours of the model phase space density (bold) and the actual data for time interval 2 .

emission. In Fig. 13 we plot the model calculated growth rate for the fitted value of $T_{\perp} / T_{\|}=1.18$ (solid). The peak growth rate is weak, about $0.014 \% f_{\text {real }}$ for $f_{\text {real }}=776 \mathrm{~Hz}$ and $\theta=0.1^{\circ}$. The peak growth rate decreases for larger $\theta$ up to about $10^{\circ}$. Also shown is a curve for a larger, arbitrarily chosen value of the temperature anisotropy, $T_{\perp} / T_{||}=3.06$, for the higher energy pancake distribution only. As in the case of time interval 1, the growth rate is seen to increase significantly for increasing values of $T_{\perp} / T_{\|}$. The growth rate is now seen to
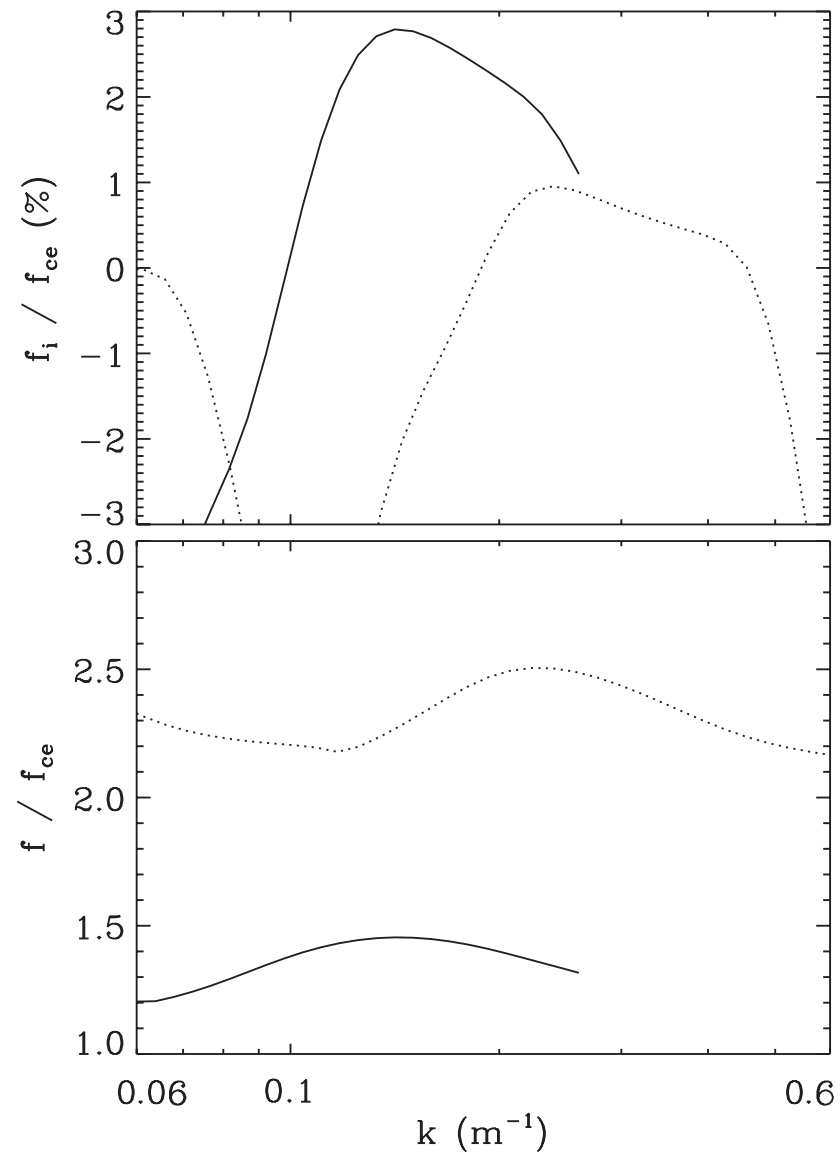

Fig. 11. Results of the growth rate analysis for the EC fundamental (solid) and first harmonic (dotted) emissions in the same format as in Fig. 6. The fundamental emission growth rate is about 3 times that of the first harmonic.

peak at $f_{i} / f_{\text {real }}=0.059 \%$ for $f_{\text {real }}=991 \mathrm{~Hz}$, and the maximum frequency is near $1300 \mathrm{~Hz}$. The wave normal angle was held constant at $0.1^{\circ}$ for the curves shown in Fig. 13. For the whistler mode emissions we estimate the observed gain to be only 25 , yielding a large path length required to produce this gain of about $4.4 \times 10^{4} \mathrm{~km}$, much larger than for interval 1 . This is consistent with the observations of only weak whistler mode emissions.

\section{Summary and conclusions}

We have examined plasma waves observed within the Saturn inner magnetosphere during two time periods of day 303 of 2005, 03:15:20-03:15:30 (interval 1) and 02:58:30-02:58:40 (interval 2). In both intervals ECH and whistler mode emission are observed but with different characteristics. For interval 1 the EC emissions are intense at the fundamental and weak at the first harmonic. The whistler mode emissions are moderately intense. For interval 2 the EC emissions are moderately intense at the fundamental and mildly intense at the 

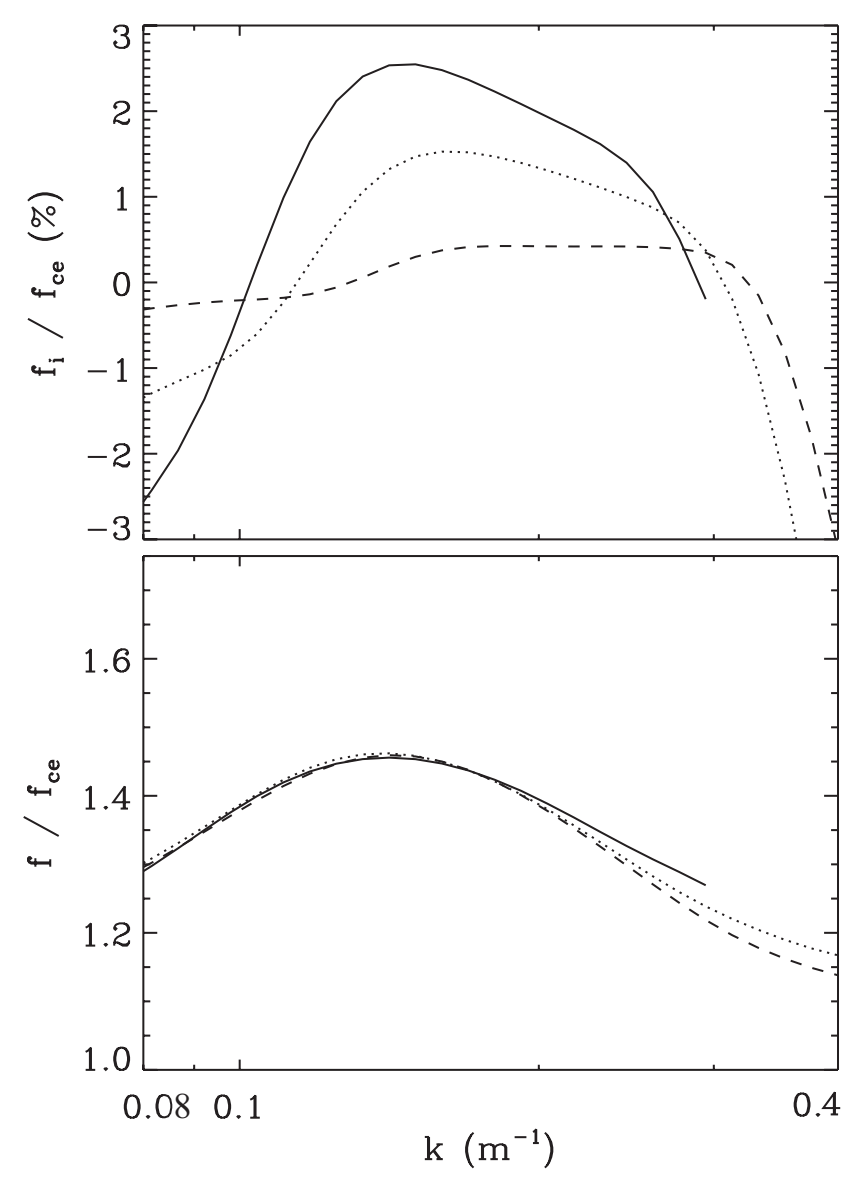

Fig. 12. Fundamental EC emission at three wave normal angles, $85^{\circ}$ (solid), $86.5^{\circ}$ (dotted), and $87.5^{\circ}$ (dashed), displaying a range of frequencies and bandwidths over which wave growth occurs.

first harmonic, while the whistler mode emissions are quite weak. By modeling the observed electron distribution for each interval we have been able to reproduce the qualitative and most of the quantitative wave growth that is observed.

The electron distribution observed by the CAPS ELS on Cassini is fit by a sum of bi-Maxwellian distributions including a cooler, less energetic $(E<1 \mathrm{keV})$ core plasma and a warmer, more energetic $(E>1 \mathrm{keV})$ distribution, each with a loss cone of about $20^{\circ}$. In addition we have included a low density cold background electron component and a cold ion background for charge neutrality. Contours of the phase space distribution function for time interval 1 suggest an electron loss cone extending to about $20^{\circ}$, the smallest pitch angle measured by ELS at the time. For time interval 2, however, the smallest pitch angle measured by ELS is about $25^{\circ}$ and no loss cone is observed. The model loss cone extends in width to about $20^{\circ}$ for both interval 1 and 2, larger than expected from only conservation of the first adiabatic invariant, but consistent with the observations. Using the linear dispersion solver, WHAMP (Ronnmark, 1982, 1983), we show that a loss cone within the cool core distribution can

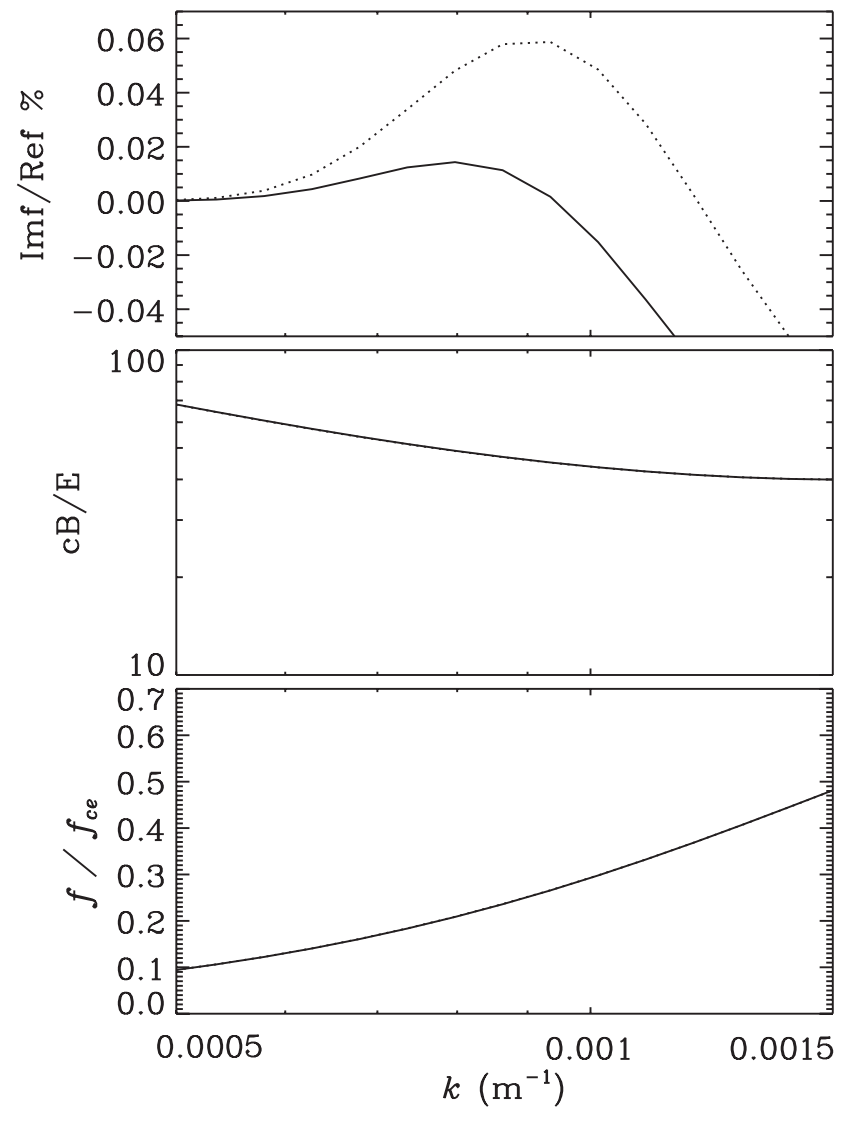

Fig. 13. The plot format is the same as Fig. 8, but the data are for interval 2 where chorus emission is observed to be weak. The whistler mode emission growth is very weak for the best fit value of $T_{\perp} / T_{\|}=1.18$ (solid) in the approximate range $500 \mathrm{~Hz}<f<900 \mathrm{~Hz}$. We also show for comparison the growth of whistler mode for $T_{\perp} / T_{\|}=3$ (dotted).

drive growth of oblique electrostatic cyclotron waves similar to those observed.

The observed whistler mode emissions can be generated by the temperature anisotropy of the warmer plasma distribution. The wave data indicate strong whistler mode emission for interval 1 and only weak emission for interval 2. Using the observed plasma distributions, the model calculations of whistler mode wave growth are consistent with a larger measured temperature anisotropy for interval 1 compared to interval 2.

\subsection{EC emissions}

The loss cone of the low-energy core electron population provides the essential free energy for the EC emission wave growth for both intervals. We have found that using a loss cone of width $\lesssim 5^{\circ}$ yields a strong first harmonic emission that is not observed. Increasing this to a loss cone width of about $20^{\circ}$, consistent with the observations which show an indication of a loss cone in Fig. 2, allows us to produce 

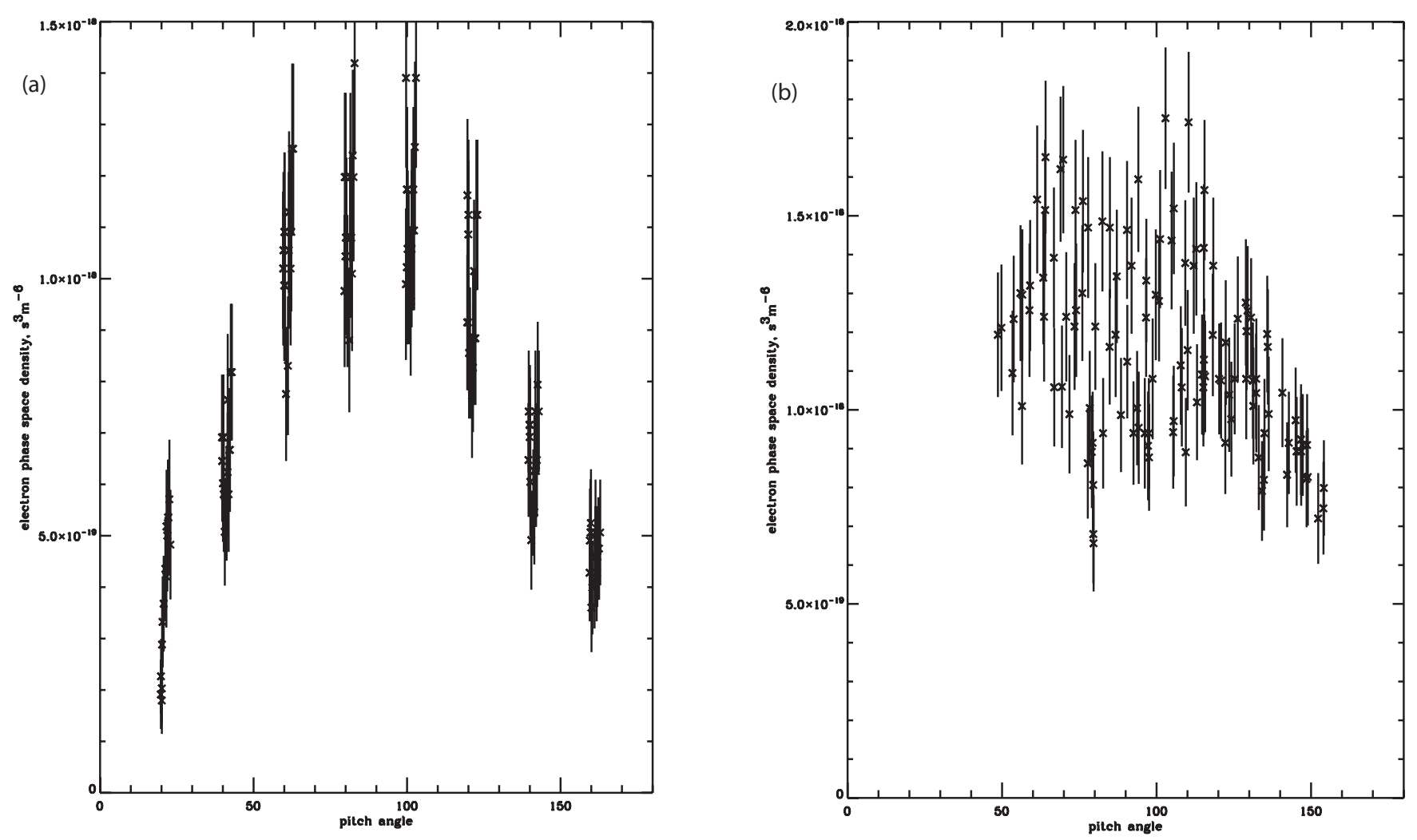

Fig. 14. The electron phase space density for electrons near $1 \mathrm{keV}$ versus pitch angle for 03:15:20-03:15:30 (a), and for the time interval 02:58:30-02:58:40 (b). The distribution for interval 1 is distinctly pancake or peaked near $90^{\circ}$, while that for interval 2 is more "butterflylike" with peaks near $65^{\circ}$ and $110^{\circ}$.

fundamental emission and much weaker first harmonic emission at interval 1 as observed. The bandwidths of the model ECH emissions substantially overlap those observed. At the fundamental the observed band extends from about $3700 \mathrm{~Hz}$ to about $5600 \mathrm{~Hz}$, with the brightest (red) emission in the range $\sim 4200 \mathrm{~Hz}$ to $5000 \mathrm{~Hz}$. If the weakest (green) emission is included the band extends from $f_{c e}(3468 \mathrm{~Hz})$ to about $5800 \mathrm{~Hz}$. The model emission extends from about $4100 \mathrm{~Hz}$ to about $5500 \mathrm{~Hz}$, so is somewhat smaller than the observed band. The first harmonic emission extends from about $8000 \mathrm{~Hz}$ to about $9600 \mathrm{~Hz}$, with slightly more intense emission ranging from about 8200 to $9000 \mathrm{~Hz}$. The model emission band extends from about $7300 \mathrm{~Hz}$ to about $9200 \mathrm{~Hz}$, and is thus shifted down in frequency by several hundred $\mathrm{Hz}$.

For interval 2 the model lower-energy, core plasma is warmer than that used in the model for interval 1 , and we have used a warmer cold electron background, but the loss cone width is the same as for interval 1 . With these parameters we are able to produce intense fundamental EC emission and moderately intense first harmonic emission as observed. During time interval 2 the more intense fundamental emission extended from about $3850 \mathrm{~Hz}$ to $5300 \mathrm{~Hz}$, while this plus a much weaker emission extended from $f_{c e}$ $(3725 \mathrm{~Hz})$ to about $6300 \mathrm{~Hz}$. The model emission extends from about $4300 \mathrm{~Hz}$ to about $5450 \mathrm{~Hz}$, so the model emission overlaps substantially the more intense emission but does not extend to the highest observed frequencies of the weaker emission. The first harmonic emission is observed to extend weakly from about $8200 \mathrm{~Hz}$ to about $9750 \mathrm{~Hz}$. The model first harmonic emission extends from about $8100 \mathrm{~Hz}$ to about $9350 \mathrm{~Hz}$, overlapping well, but about $400 \mathrm{~Hz}$ lower at the upper frequency cutoff. The discrepancies for both time intervals in the observed bandwidths compared to the model bandwidths may be explained by temporal variations of the thermal velocity and density of the plasma, and the lack of knowledge of the exact shape of the loss cone.

\subsection{Whistler mode emission}

In addition to ECH emission, both time intervals show whistler mode emission for $f<f_{c e}$. The free energy source for this emission is the warmer electron distribution with a temperature anisotropy. The whistler mode emission for interval 2 is considerably weaker than that for interval 1 . The explanation for the difference in the whistler mode emission for these two different time intervals is due to the measured temperature anisotropy in each interval. This is also clearly portrayed in the observed pitch angle distribution of 
the electrons. In Fig. 14a we plot the electron phase space density measured by ELS for electrons near $1 \mathrm{keV}$ versus pitch angle for the time interval 03:15:20-03:15:30 (interval 1), while in Fig. 14b we plot the same for the time interval 02:58:30-02:58:40 (interval 2). The distribution for the later time interval (14a) is distinctly pancake or peaked near $90^{\circ}$, while that of Fig. 14b is more "butterfly-like" with peaks near $65^{\circ}$ and $110^{\circ}$. Since the growth rate of the whistler mode is strongly dependent on the temperature anisotropy, the distribution of Fig. 14a produces the stronger wave growth. We note that for time interval 1 the model whistler mode emission gain is somewhat low for the fitted value of temperature anisotropy. One explanation for this discrepancy could be a source region of whistler emission that is remote from the observations as well as temporal effects of the measured electron phase space distribution. The success in explaining the salient features of the wave observations is encouraging, and suggests an understanding of the basic physical processes for the region outside plasma injection regions in the inner Saturn magnetosphere.

Acknowledgements. The authors thank J. Hospodarsky and K. Kurth for clerical assistance. This work was supported by Jet Propulsion Laboratory Contract 1279973 to the University of Iowa. O. Santolik acknowledges additional support of the NSF award 030719/KONTAKT ME 842 and GAAV grant IAA 301120601.

Topical Editor I. A. Daglis thanks J. Burch and another anonymous referee for their help in evaluating this paper.

\section{References}

André, N., Persoon, A. M., Goldstein, J., Burch, J. L., Louarn, P., Lewis, G. R., Rymer, A. M., Coates, A. J., Kurth, W. S., Sittler, E. C., Thomsen, M. F., Crary, F. J., Dougherty, M. K., Gurnett, D. A., and Young, D. T.: Magnetic signatures of plasma-depleted flux tubes in the Saturnian inner magnetosphere, Geophys. Res. Lett., 34, L14108, doi:10.1029/2007GL030374, 2007.

Ashour-Abdalla, M. and Kennel, C. F.: Nonconvective and convective electron cyclotron harmonic instabilities, J. Geophys. Res., 83(A4), 1531-1543, 1978.

Burch, J. L., Goldstein, J., Hill, T. W., Young, D. T., Crary, F. J., Coates, A. J., Andre, N., Kurth, W. S., and Sittler Jr., E. C.: Properties of local plasma injections in Saturn's magnetosphere, Geophys. Res. Lett., 32, L14S02, doi:10.1029/2005GL022611, 2005.

Burch, J. L., Goldstein, J., Lewis, W. S., Young, D. T., Coates, A. J., Dougherty, M. K., and Andre, N.: Tethys and Dione as sources of outward-flowing plasma in Saturn's magnetosphere, Nature, 447, 833-835, 2007.

Connerney, J. E. P., Acuna, M. H., and Ness, N. F.: Currents in Saturn's magnetosphere, J. Geophys. Res., 88, 8779-8789, 1983.

Glauert, S. A. and Horne, R. B.: Calculation of pitch angle and energy diffusion coefficients with the PADIE code, J. Geophys. Res., 110, A04206, doi:10.1029/2004JA010851, 2005.

Gurnett, D. A., Kurth, W. S., Kirchner, D. L., Hospodarsky, G. B., Averkamp, T. F., Zarka, P., Lecacheux, A., Manning, R., Roux, A., Canu, P., Cornilleau-Wehrlin, N., Galopeau, P., Meyer, A.,
Bostrom, R., Gustafsson, G., Wahlund, J.-E., Aahlen, L., Rucker, H. O., Ladreiter, H. P., Macher, W., Woolliscroft, L. J. C., Alleyne, H., Kaiser, M. L., Desch, M. D., Farrell, W. M., Harvey, C. C., Louarn, P., Kellogg, P. J., Goetz, K., and Pedersen, A.: The Cassini radio science investigation, Space Sci. Rev., 114, 395-463, 2004.

Gurnett, D. A., Kurth, W. S., Hospodarsky, G. B., Persoon, A. M., Averkamp, T. F., Cecconi, B., Lecacheux, A., Zarka, P., Canu, P., Cornilleau-Wehrlin, N., Galopeau, P., Roux, A., Harvey, C., Louarn, P., Bostrom, R., Gustafsson, G., Wahlund, J.-E., Desch, M. D., Farrell, W. M., Kaiser, M. L., Goetz, K., Kellogg, P. J., Fischer, G., Ladreiter, H.-P., Rucker, H., Alleyne, H., and Pedersen, A.: Radio and plasma wave observations at Saturn from Cassini's approach and first orbit, Science, 307(5713), 12551259, 2005.

Hill, T., Dessler, A., and Maher, L.: Corotating Magnetospheric Convection, J. Geophys. Res., 86(A11), 9020-9028, 1981.

Hill, T. W., Rymer, A. M., Burch, J. L., Crary, F. J., Young, D. T., Thomsen, M. F., Delapp, D., Andre, N., Coates, A. J., and Lewis, G. R.: Evidence for rotationally driven plasma transport in Saturn's magnetosphere, Geophys. Res. Lett., 32, L14S10, doi:10.1029/2005GL022620, 2005.

Horne, R. B. and Thorne, R. M.: Electron pitch angle diffusion by electrostatic electron cyclotron harmonic waves: The origin of pancake distributions, J. Geophys. Res., 105(A3), 5391-5402, 2000.

Horne, R. B. and Thorne, R. M.: Relativistic electron acceleration and precipitation during resonant interactions with whistler-mode chorus, Geophys. Res. Lett., 30(10), 1527, doi:10.1029/2003GL016973, 2003.

Horne, R. B., Thorne, R. M., Meredith, N. P., and Anderson, R. R.: Diffuse auroral electron scattering by electron cyclotron harmonic and whistler mode waves during an isolated substorm, J. Geophys. Res., 108(A7), 1290, doi:10.1029/2002JA009736, 2003.

Hospodarsky, G. B., Averkamp, T. F., Kurth, W. S., Gurnett, D. A., Menietti, J. D., Santolik, O., and Dougherty, M. K.: Observations of chorus at Saturn using the Cassini radio and plasma wave instrument, J. Geophys. Res., in press, doi:10.1029/2008JA013237, 2008.

LeDocq, M. J., Gurnett, D. A., and Hospodarsky, G. B.: Chorus source locations from VLF Poynting flux measurements with the Polar spacecraft, Geophys. Res. Lett., 25, 4063-4066, 1998.

Louarn, P., Kurth, W. S., Gurnett, D. A., Hospodarsky, G. B., Persoon, A. M., Cecconi, B., Lecacheux, A., Zarka, P., Canu, P., Roux, A., Rucker, H. O., Farrell, W. M., Kaiser, M. L., Andre, N., Harvey, C., and Blanc, M.: Observation of similar radio signatures at Saturn and Jupiter: Implications for the magnetospheric dynamics, Geophys. Res. Lett., 34, L20113, doi:10.1029/2007GL030368, 2007.

Means, J. D.: Use of the three-dimensional covariance matrix in analyzing the polarization properties of plane waves, J. Geophys. Res., 77, 5551-5559, 1972.

Menietti, J. D., Santolik, O., Rymer, A. M., Hospodarsky, G. B., Persoon, A. M., Gurnett, D. A., Coates, A. J., and Young, D. T.: Analysis of plasma waves observed within local plasma injections seen in Saturn's magnetosphere, J. Geophys. Res., 113, A05213, doi:10.1029/2007JA012856, 2008.

Meredith, N. P., Cain, M., Horne, R. B., Thorne, R. M., Summers, 
D., and Anderson, R. R.: Evidence for chorus-driven electron acceleration to relativistic energies from a survey of geomagnetically disturbed periods, J. Geophys. Res., 108(A6), 1248, doi:10.1029/2002JA009764, 2003.

Nunn, D., Omura, Y., Matsumoto, H., Nagano, I., and Yagitani, S.: The numerical simulation of VLF chorus and discrete emissions observed on the Geotail satellite using a Vlasov code, J. Geophys. Res., 102(A12), 27 083-27 097, 1997.

Omura, Y., Nunn, D., Matsumoto, H., and Rycroft, M. J.: A review of observational theoretical, and numerical studies of VLF triggered emissions, J. Atmos. Terr. Phys., 53, 351-368, 1991.

Parrot, M., Santolik, O., Cornilleau-Wehrlin, N., Maksimovic, M., and Harvey, C. C.: Source location of chorus emissions observed by Cluster, Ann. Geophys., 21, 473-480, 2003,

http://www.ann-geophys.net/21/473/2003/.

Ronnmark, K.: WHAMP-waves in a homogeneous, anisotropic, multi-component plasma, Rep. 179, Kiruna Geophys. Inst., Sweden, 1982.

Ronnmark, K.: Computation of the dielectric tensor of a Maxwellian plasma, Plasma Phys., 25, 699-701, 1983.

Rymer, A. M., Mauk, B. H., Hill, T. W., Paranicas, C., Andre, N., Sittler Jr., E. C., Mitchell, D. G., Smith, H. T., Johnson, R. E., Coates, A. J., Young, D. T., Bolton, S. J., Thomsen, M. F., and Dougherty, M. K.: Electron sources in Saturn's magnetosphere, J. Geophys. Res., 112, A02201, doi:10.1029/2006JA012017, 2007.
Rymer, A. M., Mauk, B. H., Hill, T. W., Paranicas, C., Mitchell, D. G., Coates, A. J., and Young, D. T.: Electron circulation in Saturn's magnetosphere, J. Geophys. Res., 113, A01201, doi:10.1029/2007JA012589, 2008.

Santolik, O. and Parrot, M.: The wave distribution function in a hot magnetospheric plasma: The direct problem, J. Geophys. Res., 101(A5), $10639-10651,1996$.

Santolik, O., Gurnett, D. A., and Pickett, J. S.: Multipoint investigation of the source region of storm-time chorus, Ann. Geophys., 22, 2555-2563, 2004, http://www.ann-geophys.net/22/2555/2004/.

Young, D. T., Berthelier, J. J., Blanc, M., Burch, J. L., Coates, A. J., Goldstein, R. R., Grande, M., Hill, T. W., Johnson, R. E., Kelha, V., McComas, D. J., Sittler, E. C., Svenes, K. R., Szegö, K., Tanskanen, P., Ahola, K., Anderson, D., Bakshi, S., Baragiola, R. A., Barraclough, B. L., Black, R. K., Bolton, S., Booker, T., Bowman, R., Casey, P., Crary, F. J., Delapp, D., Dirks, G., Eaker, N., Funsten, H., Furman, J. D., Gosling, J. T., Hannula, H., Holmlund, C., Huomo, H., Illiano, J. M., Jensen, P., Johnson, M. A., Linder, D. R., Luntama, T., Maurice, S., McCabe, K. P., Mursula, K., Narheim, B. T., Nordholt, J. E., Preece, A., Rudzki, J., Ruitberg, A., Smith, K., Szalai, S., Thomsen, M. F., Viherkanto, K., Vilppola, J., Vollmer, T., Wahl, T. E., Wüest, M., Ylikorpi T., and Zinsmeyer, C.: Cassini plasma spectrometer investigation, Space Sci. Rev., 114, 1-112, 2004. 